\title{
Hemiballismus and normal pressure hydrocephalus as long-term sequelae following a hemispherectomy for intractable epilepsy in a man with a learning disability
}

\author{
Joseph Joyce ${ }^{1}$ and Shoumitro Deb $^{2}$ \\ ${ }^{1}$ Ely Hospital, Cardiff, UK, ${ }^{2}$ Psychological Medicine, University of Wales College of \\ Medicine, University of Wales, Cardiff, UK \\ Correspondence to: Shoumitro Deb, Clinical Senior Lecturer and Honorary \\ Consultant in Neuropsychiatry, Psychological Medicine, University of Wales College \\ of Medicine, University Hospital of Wales, Heath Park, Cardiff, CF4 4XN, UK
}

\begin{abstract}
We report here a case of a 48-year-old gentleman with mild/moderate learning disability who developed late complications of hemispherectomy 28 years following the operation. The original operation was performed to treat intractable epilepsy (both generalized tonic/clonic and complex partial seizures) in an 8-year-old boy. After a 28 year seizure-free period, the patient developed cognitive decline, seizures, hemiballismus and a subacute confusional state, possibly related to normal pressure hydrocephalus. A ventriculo-peritoneal shunt operation, along with treatment with high doses of sulpiride, brought improvement in both mental state and hemiballismus.
\end{abstract}

Keywords: Hemispherectomy - Hydrocephalus, normal pressure - Cerebral hemidecortication

\section{INTRODUCTION}

The operation of hemispherectomy (or cerebral hemidecortication) consists of the removal of the cortex of one cerebral hemisphere plus a variable portion of the basal ganglia underneath. The operation was first reported in the treatment of epilepsy in a patient with infantile hemiplegia by McKenzie in 1938, at an oral presentation, and the first series of such operations for infantile hemiplegia was reported by Krynauw (1950). From about 1950 until 1970 many such operations were performed. The operation produced a substantial relief of epileptic seizures in a high percentage of the patients undergoing it, providing the pre-existing damage was largely or entirely confined to one cerebral hemisphere. Wilson (1970) reported a follow-up of 50 patients who had the operation performed up to 19 years earlier and he found that seizures were completely or substantially relieved in more than $80 \%$ of the patients. Usually the hemiparesis was not worsened by the operation but all patients developed a homonymous hemianopia after the operation. Hemispherectomy did not usually depress intellectual function and it sometimes appeared to enhance intellectual function (Krynauw, 1950; Wilson, 1970; Smith and Sugar, 1975). Mild mental handicap was not a contra-indication to the procedure.

Early complications of the operation were well known and included haemorrhage, infection, fatal brain stem displacement and hydrocephalus of the cavity and remaining hemisphere (Krynauw, 1950; Wilson, 1970; Goodman, 1986). Serious late complications have been documented in the literature since the mid 1960s. In Wilson's series, 11 (22\%) patients died more than five years after operation in addition to $5(10 \%)$ who died within the first two years. The principal delayed complications of hemispherectomy are haemorrhage, hydrocephalus and haemosiderosis, which are documented as occurring from one year to 24 years postoperatively (Wilson, 1970; Adams, 1983; Rasmussen, 1983; Goodman, 1986).

\section{CASE REPORT}

Mr J N was the first born of three siblings in $1947 . \mathrm{He}$ was a full-term baby who was delivered after a three- 
hour labour. There was no family history of mental handicap, epilepsy or psychiatric illness. At the age of 11 months he developed a fever of $101^{\circ} \mathrm{F}$ and epileptic seizures, one of which lasted for seven hours. He became comatose and, about three days later, he developed a rash of a measles type. He was diagnosed as having a viral encephalitis and, on his recovery from the acute illness, he had a left hemiparesis. By the age of two years he was able to walk unaided but he was noted to have a left homonymous hemianopia. At the age of two and a half years he developed recurrent epileptic seizures of two types, simple partial seizures and generalized tonic clonic seizures. By the age of four and a half he was noted to have learning disabilities but he had learned to speak. At the age of six years he was noted to have poor concentration and to have developed behavioural problems. By the age of eight years his epilepsy was not responding to phenobarbitone and he was having up to 30 seizures per day. He was said to have scored 67 on an IQ test during his early childhood but, on psychometric testing just

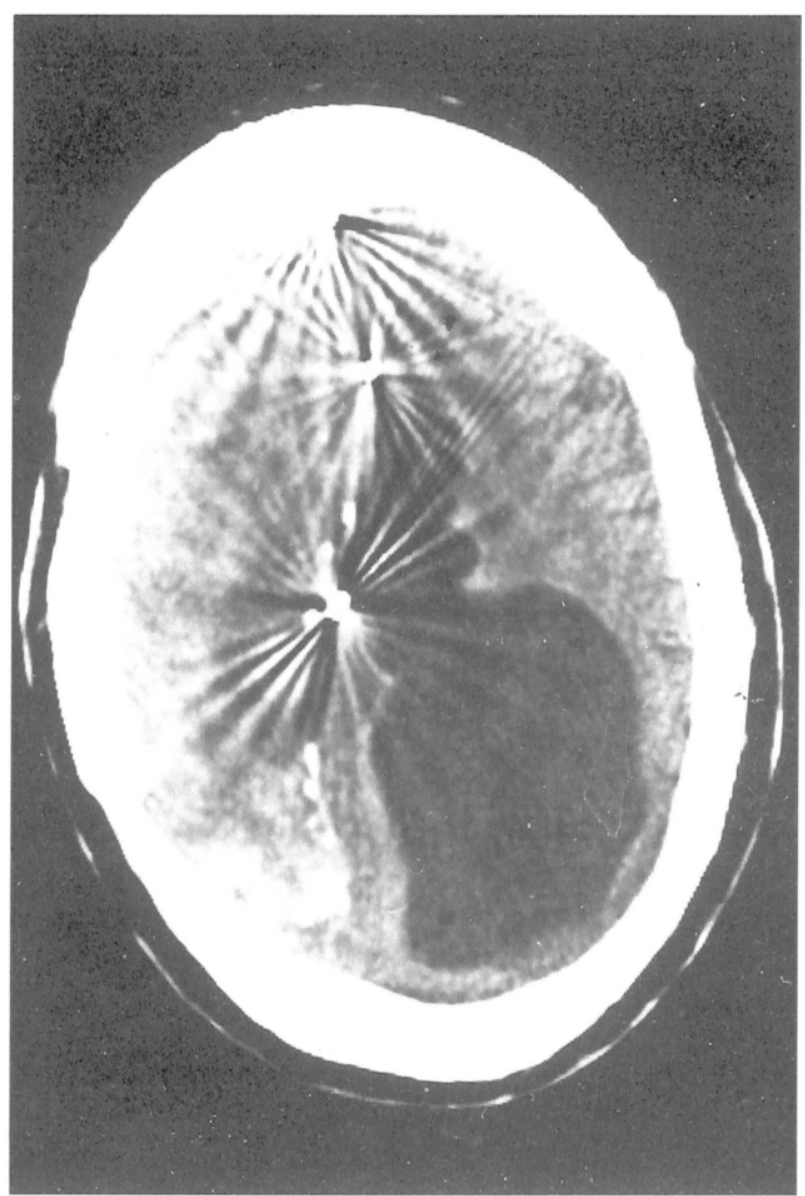

FIG. 1: Cranial CT scan of the patient. before surgery, he was given an overall IQ score of 50-55 (Terman Merrill and Merrill Palmer scales). His mental age on verbal tests was, at most, four and a half years (verbal IQ 60) but visuo-spatial tests gave an IQ of below 40. His single best subscale score was in vocabulary on which he scored at the six-year-old level.

In 1955 (aged eight) he underwent a right sided hemispherectomy at the National Hospital, Queen Square, London. There was a complete remission of his epileptic seizures and his antiepileptic drugs were withdrawn. There were no further reports of behavioural problems. He lived with his parents and, in adult life, attended an adult training centre. His learning disability persisted but he is reported to have enjoyed life and was said to be very sociable and likeable.

In 1985, 30 years after the hemispherectomy and at the age of 38 , he presented to a neurologist with a two year history of slurred speech, muttering and involuntary movements of the limbs of his right side. A CT scan carried out at that time showed a dilated left lateral ventricle and a small right cranium. Observation

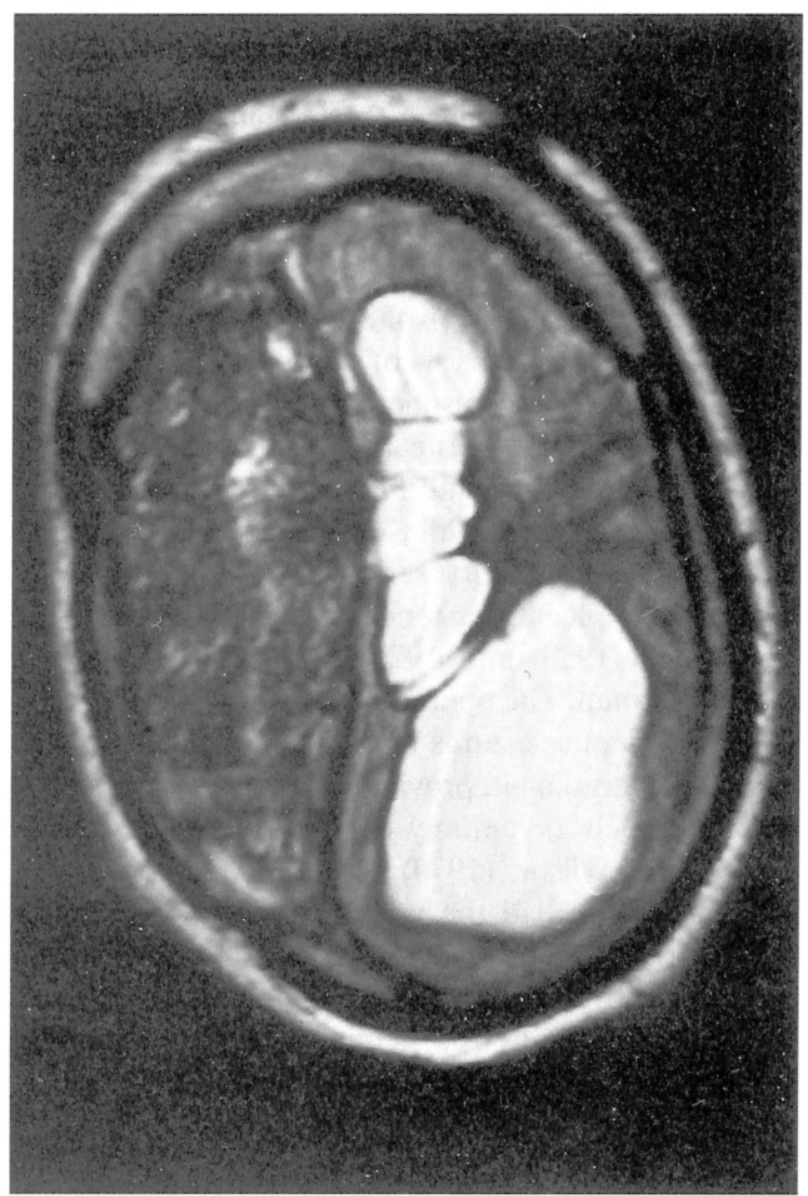

FIG. 2: Cranial MRI scan of the patient.

44 Behavioural Neurology . Vol 10 . 1997 
of the movements showed them to be hemiballismus. $\mathrm{He}$ was treated with sulpiride $2,400 \mathrm{mg}$ daily, with dramatic improvement. He moved out of home to live in a group home with four other people with learning disabilities and received continuous staff support.

Four years later (aged 42) there was evidence of memory loss and a recurrence of his epilepsy with generalized tonic clonic seizures and complex partial seizures. Psychometric assessment in 1991 on WAIS-R showed a verbal IQ of 64 with performance IQ of less than 54 (i.e., below the lowest limit of the scale) with marked impairment of visuo-spatial skills. The visual object and space perception battery was administered and showed JN to have good space perception but there were marked difficulties with more complex stimuli. JN was able to draw simple shapes but not more complex shapes, such as a house, cat or stick man. There was severe memory impairment and severe impairment of new learning abilities. There was a severe disorder of orientation, attention, calculation, recognition, naming learning and verbal fluency on the Mini Mental State and MEAMS test (Middlesex Elderly Assessment of Mental State).

His epilepsy was treated with phenytoin $(250 \mathrm{mg}$ per day) and sodium valproate (1000 mg per day). In 1992, he suffered a three-day episode of slurred speech, weakness on one side of his body and confusion. This was diagnosed as a stroke and he was treated with aspirin. Over the next six months, he suffered from intermittent confusional states with urinary and faecal incontinence, ataxia and frequent falls. There was a general decline in his abilities and he was admitted to hospital for investigation to eliminate treatable causes of dementia. A CT scan showed no change from 1985. $\mathrm{He}$ suffered a prolonged tonic clonic seizure. He was referred to the neurosurgical team who found his intracranial pressure to be normal. An MRI scan showed obstruction to CSF flow with marked dilation of the left lateral ventricle. Neuroimaging findings are shown in Fig. 1 (CT scan), 2 (MRI scan) and 3 (SPECT scan). A left-sided ventriculo-peritoneal shunt was performed with marked improvement in his mental state in that he was brighter in spirits and showed more interest in his surroundings. Although he continued to have epileptic seizures, the frequency was reduced to, on average, once every four to six weeks. He is able to walk with the help of two people. $\mathrm{He}$ is currently living in a nursing home as his deterioration in the two years between 1991 and 1993 was such that he could not return to live in his home. He is taking antiepileptic drugs and he is taking sulpiride to control his hemiballismus.

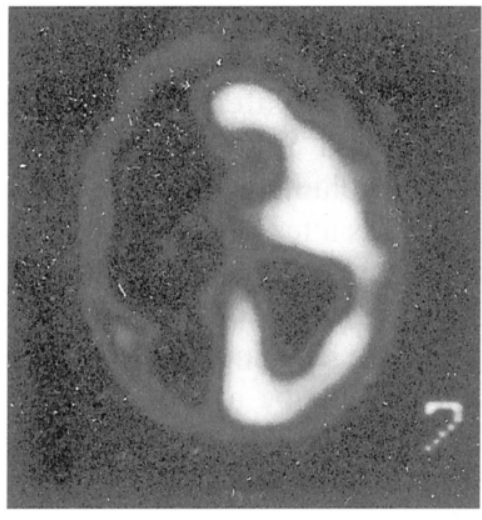

FIG. 3: SPECT brain scan of the patient.

\section{DISCUSSION}

This man is probably one of the 50 cases described by Wilson (1970). He had 28 years of epilepsy-free life from the time of his operation before the first complication developed. He then had another four years of freedom from epilepsy with treatment of his hemiballismus before he needed to take antiepileptic drugs again. Superficially, he experienced a good quality of life but he obviously suffered the handicaps of learning disabilities, hemiparesis and homonymous hemianopia. In the study reported by Lindsay et al. (1987), two of the six patients who were free of seizures for ten years after hemispherectomy developed recurrence of seizures within the follow-up period.

Hydrocephalus is a treatable long-term complication of hemidecortication (Wilson, 1970; Rasmussen 1983). The pathogenesis of this complication was first described by Oppenheimer and Griffiths (1966). It may present with pressure effects, such as headache and vomiting, following a rapid change due to bleeding or it may present with intermittent episodes of acute confusion, ataxia and urinary incontinence, as in normal pressure hydrocephalus. Modifications to the operation of cerebral hemidecortication have been proposed to lessen the long-term risks of the development of hydrocephalus (Adams, 1983; Rasmussen, 1983; Goodman, 1986). In retrospect, this man's hydrocephalus probably caused symptoms from 1989, four years before he was referred to neurosurgery, while he still had a good quality of life. A successful shunt operation at that stage could have prevented his deterioration and it may have brought his epilepsy under control again (Lindsay et al., 1987).

Hemiballismus is a movement disorder in which the affected person has involuntary large amplitude movements of the limbs of one side of the body. Damage to 
the subthalamic nucleus on the contralateral side is said to be the cause of most cases. The damage may be caused by a range of different illnesses (Shannon, 1990; Provenzale and Schwarzschild, 1994). Wilson (1970) mentions involuntary movements (but does not specify what type) as a feature of intracranial bleeding in patients after hemispherectomy. Thalamotomy and head injury are mentioned as known causes of hemiballismus in the literature. Our patient's hemiballismus was assumed to be a late complication of his surgery and was treated with sulpiride in high dosage. Klawans et al. (1976) describe 11 cases of hemiballismus which were treated with either chlorpromazine or haloperidol. Dewey and Jankovic (1989) list 22 different types of drugs which have been used to treat hemiballismus. None of these authors mention sulpiride as a treatment for hemiballismus but low doses (150 mg daily) of sulpiride have been used with good effect by Hanaoka et al. (1990).

Most people who have had a hemispherectomy have some degree of learning disability for the rest of their lives (Vargha-Khadem and Polkey, 1992) with occasional notable exceptions (Smith and Sugar, 1975). A deterioration in cognitive functioning in these people may be due to a treatable long-term complication of the original surgery and it is obviously important to diagnose this early in the course of the deterioration.

\section{Acknowledgements}

The authors wish to thank Sue Collyer and Ceri Buckler for patiently typing the manuscript.

\section{REFERENCES}

Adams CBT (1983) Hemispherectomy - a modification. Journal of Neurology, Neurosurgery and Psychiatry 46, 617-619.

Dewey, RB and Jankovic J (1989) Hemiballism Hemichorea clinical and pharmacologic findings in 21 patients. Archives of Neurology 46, 862-867.

Goodman R (1986) Hemispherectomy and its alternatives in the treatment of intractable epilepsy in patients with infantile hemiplegia. Developmental Medicine and Child Neurology 28, 251-258.

Hanaoka Y, Ohi T and Matsukura S (1990) A case of hemiballism successfully treated by sulpiride, caused by lesions of the striatum. Rinsho Shinkeigaku 30, 774-776.

Klawans HL, Moses H, Nausieda PA, Bergen D and Weiner WJ (1976) Treatment and prognosis of hemiballismus. New England Journal of Medicine 295, 1348-1350.

Krynauw RA (1950) Infantile hemiplegia treated by removing one cerebral hemisphere. Journal of Neurology, Neurosurgery and Psychiatry 13, 243-267.

Lindsay J, Ounstead C and Richards P (1987) Hemispherectomy for childhood epilepsy: a 36 year study. Developmental Medicine and Child Neurology 29, 592-600.

McKenzie KG (1938) The present status of a patient who has a right cerebral hemisphere removed. Journal of the American Medication Association 111, 168.

Oppenheimer DR and Griffiths HB (1966) Persistent intracranial bleeding as a complication of hemispherectomy. Journal of Neurology, Neurosurgery and Psychiatry 29, 229-240.

Provenzale JM and Schwarzschild MA (1994) RadiologicClinical Correlation: Hemiballismus. American Journal of Neuroradiology 15, 1377-1382.

Rasmussen T (1983) Hemispherectomy for seizures revisited. Canadian Journal of Neurological Sciences 10 , $71-78$.

Shannon KM (1990) Hemiballismus. Clinical Neuropharmacology 13, 413-425.

Smith A and Sugar O (1975) Development of above normal language and intelligence 21 years after left hemispherectomy. Neurology 25, 813-818.

Vargha-Khadem F and Polkey CE (1992) A review of cognitive outcome after hemidecortication in humans. Advances in Experimental Medicine and Biology 325, 137-151.

Wilson PJE (1970) Cerebral hemispherectomy for infantile hemiplegia: a report of 50 cases. Brain 93, 147-180.

(Received 22 July 1996; accepted 9 January 1997) 


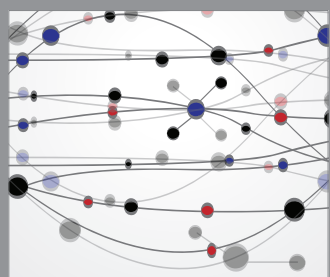

The Scientific World Journal
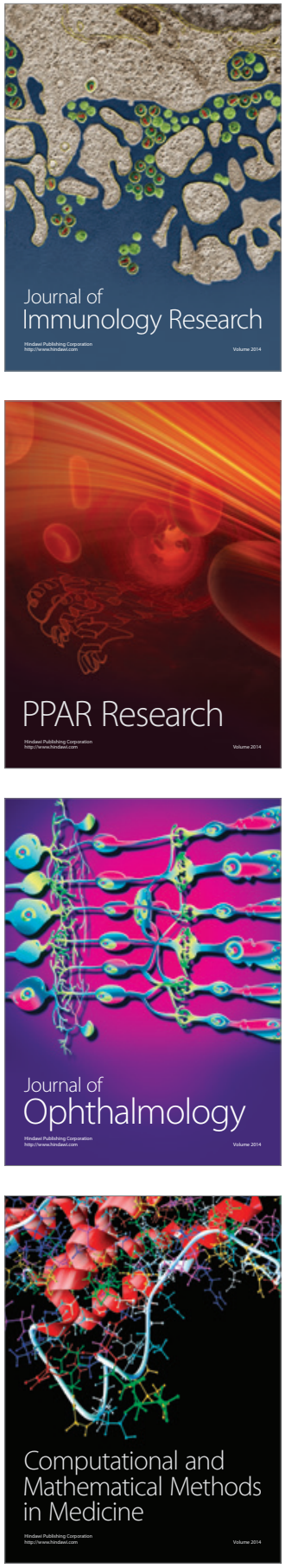

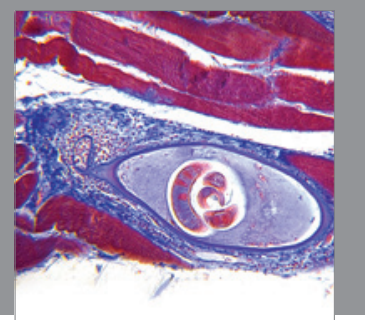

Gastroenterology

Research and Practice
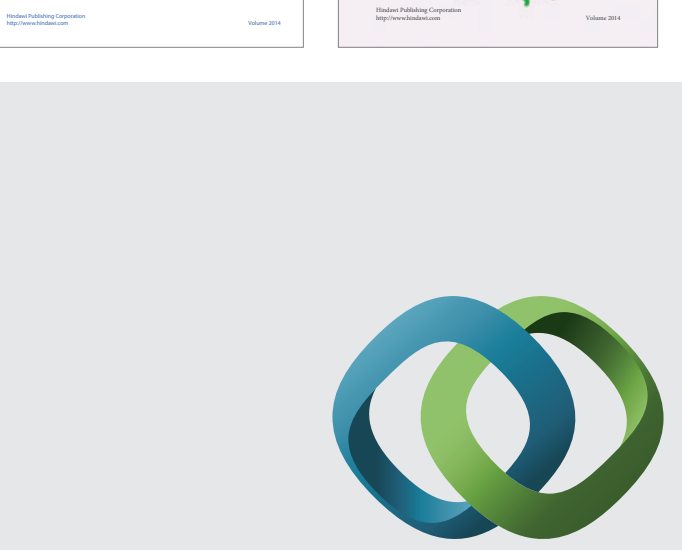

\section{Hindawi}

Submit your manuscripts at

http://www.hindawi.com
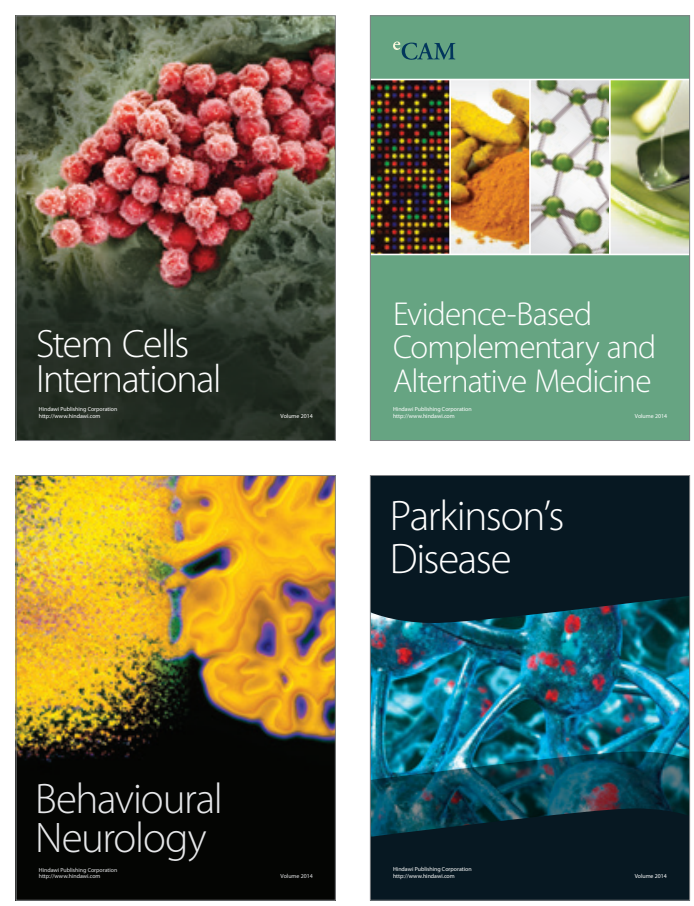

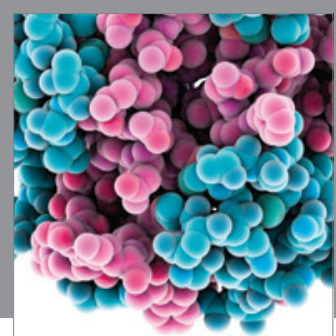

Journal of
Diabetes Research

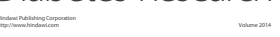

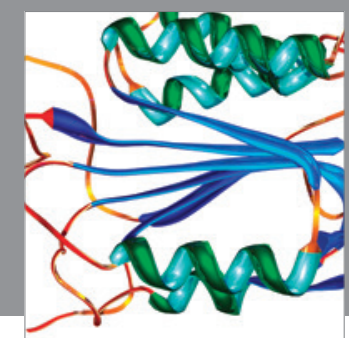

Disease Markers
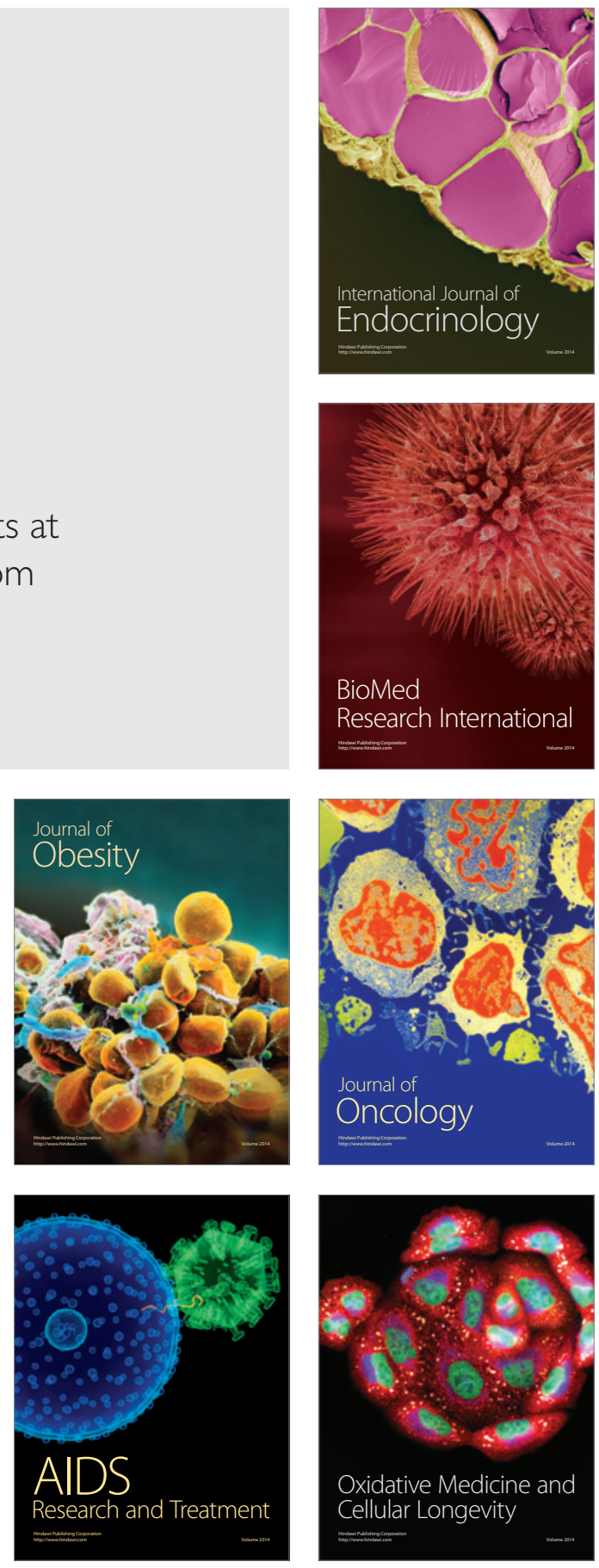